Gazi University
Journal of Science
http://dergipark.gov.tr/gujs

\title{
Actions of Double Group-Groupoids and Covering Morphism
}

\author{
Serap DEMIR* (10), Osman MUCUK \\ Erciyes University, Faculty of Science, Department of Mathematics, 38039, Kayseri, Turkey
}

\author{
Highlights \\ - Covering morphism of double groupoids derived by action double groupoid was considered. \\ - Action double group-groupoid on a group-groupoid was characterized. \\ - Covering morphism of double group-groupoids was obtained. \\ - A categorical equivalence was proved.
}

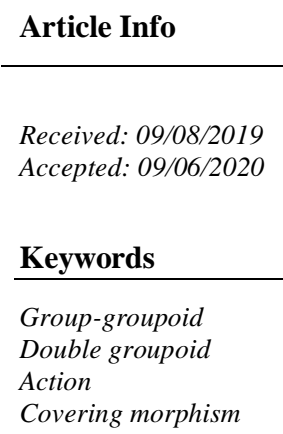

\section{INTRODUCTION}

The concept of actions and coverings of groupoids have a significant role in the utilization of groupoids (see [1] and [2]). It is familiar that for a certain groupoid $G$, the category $\operatorname{GpdAct}(G)$ of groupoid actions of $G$ on sets and the category $G p d C o v / G$ of covering morphisms of $G$ are equivalent. Topological version of this equivalence was given in [3]. An associated categorical equivalence was proved in [4, Proposition 3.1] for the case $G$ is a group-groupoid used under the name 2-group [5] and $G$-groupoids [6]. For different generalizations of this result see [7-10].

Double groupoids defined by Ehresmann in $[11,12]$ as internal groupoids in the category of groupoids are useful to calculate the fundamental groupoids of topological spaces [13]. By [14] double groupoids are categorically equivalent to crossed modules defined by Whitehead in $[15,16]$ and this categorical equivalence was characterized for group-groupoids in [17]. Quotient and normal double groupoids were characterized in [18]. In [19, Theorem 1.7] it was showed that horizontal actions and horizontal action morphisms of a double Lie groupoid are equivalent.

In this paper, for the convenience of the presentation we introduce some preliminaries on double groupoids, action of a double groupoid on a groupoid, covering groupoid and some related results. Then we extend these notions to double group-groupoids. More precisely, we define the concept of covering morphism for double group-groupoids. Finally, we prove a categorical equivalence between horizontal actions and covering morphisms associated with horizontal actions of a double group-groupoi. 


\section{PRELIMINARIES ON GROUPOIDS AND ACTIONS}

A category whose all arrows are isomorphism is called a groupoid. (see [1] and [20] for more details on groupoids). So a groupoid $G$ base on $G_{0}$ usually denoted by $G$ or $\left(G, G_{0}\right)$, has a set $G$ of arrows or elements and a set $G_{0}$ of objects with source and target maps $d_{0}, d_{1}: G \rightarrow G_{0}$ and object inclusion map $\varepsilon: G_{0} \rightarrow G$ satisfying the property $d_{0} \varepsilon=d_{1} \varepsilon=1_{G_{0}}$. For $x \in G_{0}$ the morphism $\varepsilon(x)$ acts as the identity and it is denoted by $1_{x}$. An associative partial composition of $G$ is $G * G \rightarrow G,(g, h) \mapsto g \circ h$, where $G^{*} G$ is $\left\{(g, h) \in G^{*} G \mid d_{1}(g)=d_{0}(h)\right\}$. The composite $g \circ h$ for $g, h \in G$ exists whenever $d_{1}(g)=d_{0}(h)$. Here $d_{0}(g \circ h)=d_{0}(g)$ and $d_{1}(g \circ h)=d_{1}(h)$. Each element $g$ has an inverse $g^{-1}$ with $d_{0}\left(g^{-1}\right)=d_{1}(g), d_{1}\left(g^{-1}\right)=d_{0}(g), \quad g \circ g^{-1}=\varepsilon\left(d_{0}(g)\right), g^{-1} \circ g=\varepsilon\left(d_{1}(g)\right)$. The inversion map is defined to be $G \rightarrow G, g \mapsto g^{-1}$. The source and target, the object inclusion, the inversion maps and the partial composition are all called structural maps.

For a groupoid $G$ let $x, y \in G_{0}$. We will use $G(x, y)$ to denote the set of all arrows whose source $x$ and target $y$ and $G(x, x)$ denote the object group at $x$. As mentioned in [1], for $x \in G_{0}$ the set $\left\{a \in G \mid d_{0}(a)=x\right\}$ of arrows with source $x$ is called star of $x$ and we denote it by $S t_{G} x$.

A morphism from the groupoid $H$ to $G$ consists of two maps $f_{1}: H \rightarrow G$ and $f_{0}: H_{0} \rightarrow G_{0}$ with the property $f_{1}\left(h \circ h^{\prime}\right)=f_{1}(h) \circ f_{1}\left(h^{\prime}\right)$ when $\left(h, h^{\prime}\right) \in H^{*} H$ subject to the conditions $f_{0} d_{1}=d_{0} f_{1}$ and $f_{0} d_{0}=d_{0} f_{1}$. We write $f: H \rightarrow G$ for a groupoid morphism.

If $p: H \rightarrow G$ is a morphism and for each $y \in H_{0}$, the restriction of $p, S t_{H} y \rightarrow S t_{G} p(y)$, is bijective, then $p$ is named as a covering morphism and $H$ a covering groupoid of $G$.

We remind groupoid action on a set as follows [1, p.373].

Definition 2.1. An action of a groupoid $G$ on a set $X$ includes two functions $\omega: X \rightarrow G_{0}$ and $\varphi: X * G \rightarrow X,(x, g) \mapsto x \bullet g$ where $X * G$ is the pullback of $d_{0}$ and $\omega$ and the following properties hold

i. $\quad \omega(x \bullet g)=d_{1}(g)$ for $(x, g) \in X^{*} G$,

ii. $x \bullet\left(g_{1} \circ g_{2}\right)=\left(x \bullet g_{1}\right) \bullet g_{2}$ for $\left(g_{1}, g_{2}\right) \in G^{*} G$ and $(x, g) \in X^{*} G$,

iii. $\quad x \bullet \varepsilon(\omega(x))=x$ for $x \in X$.

Write $(X, \omega)$ for an action. A morphism $f:(X, \omega) \rightarrow\left(X^{\prime}, \omega^{\prime}\right)$ is a function $f: X \rightarrow X^{\prime}$ with $\omega^{\prime} f=\omega$ and $f(x \bullet g)=f(x) \bullet g$. So we have a category $\operatorname{GpdAct}(G)$ such that objects are actions and arrows are morphisms.

Let's given such an action. According to [1, p.374], we have a groupoid $G \ltimes \omega$ base on $X$ which is called semidirect product groupoid or action groupoid. For $x, y \in X$, arrows of $(G \ltimes \omega)(x, y)$ are $(g, x)$ where $g \in G(\omega(x), \omega(y))$ and $x \bullet g=y$. The composition is defined by 


$$
\left(g_{1}, x\right) \circ\left(g_{2}, y\right)=\left(g_{1} \circ g_{2}, x\right)
$$

under the condition $y=x \bullet g_{1}$.

The projection map $p: G \ltimes \omega \rightarrow G$ defined on base by $\omega: X \rightarrow G_{0}$ and on arrows by $(g, s) \rightarrow g$ is a covering morphism. We call this action covering morphism. Hence by this assignment we have a categorical equivalence

$$
\operatorname{GpdAct}(G) \simeq G p d \operatorname{Cov} / G
$$

For a topological treatment of this equivalence we refer the readers to [3].

A groupoid whose base and arrow sets have both group structures and the structural maps are grouphomomorphism is called group-groupoid. Here $g \circ h$ is the composition of arrows in groupoid while $g+h$ is the addition in group and write $g^{-1}$ for the inverse of $g$ in groupoid and $-g$ for the one in group. Note that the groupoid composition is a group homomorphism if and only if the following interchange rule for $g, h, k, l \in G$ hold whenever one side composition is well defined

$$
(g+h) \circ(k+l)=(g \circ k)+(h \circ l) .
$$

A morphism of groupoids preserving group addition is a morphism of group-groupoids. So group-groupoids and morphisms between them form a category GpGpd.

In the action of a group-groupoid $G$ on group $X$ we have a group homomorphism $\omega: X \rightarrow G_{0}$ and an action of groupoid $G$ on the set $X$ by $\omega$ providing that

$$
(x \bullet g)+\left(x^{\prime} \bullet g^{\prime}\right)=\left(x+x^{\prime}\right) \bullet\left(g+g^{\prime}\right) \text {. }
$$

A morphism of group-groupoid actions from $(X, \omega)$ to $\left(X^{\prime}, \omega^{\prime}\right)$ is a group homomorphism $f: X \rightarrow X^{\prime}$ which is a morphism on underlying actions of $G$. This constitutes a category $\operatorname{Gp} \operatorname{GpdAct}(G)$. For this kind of action, we have semidirect product groupoid $G \ltimes \omega$ which is a group-groupoid with group composition

$$
(g, x)+\left(g^{\prime}, x^{\prime}\right)=\left(g+g^{\prime}, x+x^{\prime}\right) .
$$

The projection map $p: G \ltimes \omega \rightarrow G$ is an object of $G p G p d C o v / G$. Then by [4, Proposition 3.1], we have the categorical equivalence stated in the next theorem.

Theorem 2.2. The categories $G p G p d C o v / G$ and $G p G p d A c t(G)$ are equivalent.

\section{ACTIONS OF DOUBLE GROUPOIDS ON GROUPOIDS}

In this part we consider the covering morphism of double groupoids derived by action double groupoid following [19].

A double groupoid has four related groupoid structures with compatible structural maps. Two of these groupoids are $H$ and $V$ with base $P$. Other two are on $S$ which are vertical groupoid base on $H$ denoted by $S_{V}$ and horizontal groupoid base on $V$ denoted by $S_{H}$. From now on the set of quadruple $(S ; H, V ; P)$ denote a double groupoid. 
For a double groupoid we will use multiplicative notation for groupoid compositions in $H$ and $1_{b}$ for the identity element in $H$ for $b \in P$. The source and target maps, object inclusion, multiplication for $H$ are $d_{0}^{H}, d_{1}^{H}: H \rightarrow P, \varepsilon^{H}: P \rightarrow H$ and $m^{H}: H^{*} H \rightarrow H$ respectively and similar notations will be used for $V$.

The horizontal groupoid structure $S_{H}$ on $S$ with base $V$ have source and target $d_{0}^{h}, d_{1}^{h}: S \rightarrow V$, object inclusion $\varepsilon^{h}: V \rightarrow S$ and partial composition $\circ_{h}: S * S \rightarrow S,\left(s_{1}, S_{2}\right) \mapsto s_{1} \circ_{h} s_{2}$. Inverse is denoted by $s^{-h}$ for $h \in H$. By a similar argument, vertical groupoid $S_{V}$ on $S$ with base $H$ have source and target $d_{0}^{v}, d_{1}^{v}: S \rightarrow H, \quad$ object inclusion $\varepsilon^{v}: H \rightarrow S$ and partial composition $\circ_{v}: S * S \rightarrow S$, $\left(s_{1}, s_{2}\right) \mapsto s_{1} \circ s_{2}$. Inverse is denoted by $s^{-v}$.

Elements of $S$ are squares with boundaries as follows.

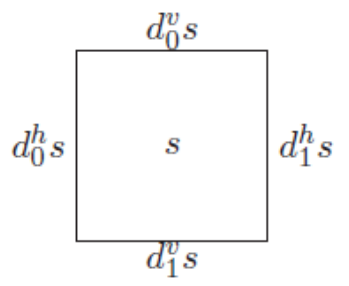

A double groupoid have the following interchange rule

$$
\left(s_{1} \circ_{h} s_{2}\right) \circ_{v}\left(s_{3} \circ_{h} s_{4}\right)=\left(s_{1} \circ_{v} s_{3}\right) \circ_{h}\left(s_{2} \circ_{v} s_{4}\right)
$$

for $s_{1}, s_{2}, s_{3}, s_{4} \in S$. Let $(S ; H, V ; P)$ and $\left(S^{\prime} ; H^{\prime}, V^{\prime} ; P^{\prime}\right)$ be two double groupoids. A morphism

$$
\phi=\left(\phi_{s}, \phi_{h}, \phi_{v}, \phi_{p}\right):\left(S^{\prime} ; H^{\prime}, V^{\prime} ; P^{\prime}\right) \rightarrow(S ; H, V ; P)
$$

consists of four maps which commute with structural maps. These constitute a category DGpd of double groupoids.

We state following construction which is originally given for Lie case appears in [19, Theorem1.7].

Definition 3.1. For a quadruple $S=(S ; H, V ; P)$ and a groupoid $G$, let $\omega: G \rightarrow V$ and $\omega_{0}: G_{0} \rightarrow P$ be morphisms. A horizontal action of double groupoid $S$ on $\left(\omega, \omega_{0}\right)$ includes actions of $S_{H}$ on $\omega: G \rightarrow V$ and $H$ on $\omega_{0}: G_{0} \rightarrow P$ in the sense of ordinary groupoid actions such that

i. $\quad d_{1}^{G}(g \bullet s)=d_{1}^{G}(g) \bullet d_{1}^{v}(s)$ and $d_{0}^{G}(g \bullet s)=d_{0}^{G}(g) \bullet d_{0}^{v}(s)$ for all $s \in S, g \in G$ satisfying $d_{0}^{h}(s)=\omega(g)$,

ii. For $s_{1}, s_{2} \in S$ and $g_{1}, g_{2} \in G$ we have

$$
\left(g_{1} g_{2}\right) \bullet\left(s_{1} \circ s_{2}\right)=\left(g_{1} \bullet s_{1}\right) \circ_{v}\left(g_{2} \bullet s_{2}\right),
$$

iii. For all $h \in H$ and $x \in G_{0}$ with $d_{0}^{H}(h)=\omega_{0}(x)$ we have $1_{x} \bullet \varepsilon^{v}(h)=1_{h x}$. 
There is also similar vertical action of double groupoid that consists of actions of $S_{V}$ on $\omega: G \rightarrow H$ and of $V$ on $\omega_{0}: G_{0} \rightarrow P$.

Denote such a horizontal action by $\left(G,\left(\omega, \omega_{0}\right)\right)$ and define $f:\left(G,\left(\omega, \omega_{0}\right)\right) \rightarrow\left(G^{\prime},\left(\omega^{\prime}, \omega_{0}^{\prime}\right)\right)$ between such actions to be consisting of groupoid homomorphisms $f: G \rightarrow G^{\prime}$ and $f_{0}: G_{0} \rightarrow G_{0}^{\prime}$ with the properties $f(g \bullet s)=f(g) \bullet s$ and $f_{0}(x \bullet h)=f_{0}(x) \bullet h$ such that the equalities $\omega^{\prime} f=\omega$ and $\omega_{0}^{\prime} f_{0}=\omega_{0}$ hold. Hence for given a double groupoid $S$ wehavea category denoted by $\operatorname{DGpdAct}_{H}(S)$.

Consider given a horizontal action of S on $\left(\omega, \omega_{0}\right)$. Then we have a quadruple $\left(S_{H} \ltimes \omega ; H \ltimes \omega_{0}, G ; G_{0}\right)$ denoted by $S \ltimes\left(\omega, \omega_{0}\right)$ and called action double groupoid with respect to horizontal action of $S$ on $\left(\omega, \omega_{0}\right)$.

Remark that we have projections $(c, \omega): S \ltimes \omega \rightarrow G$ and $\left(c_{0}, \omega_{0}\right): H \ltimes \omega_{0} \rightarrow G_{0}$ which are covering morphisms of ordinary groupoids.

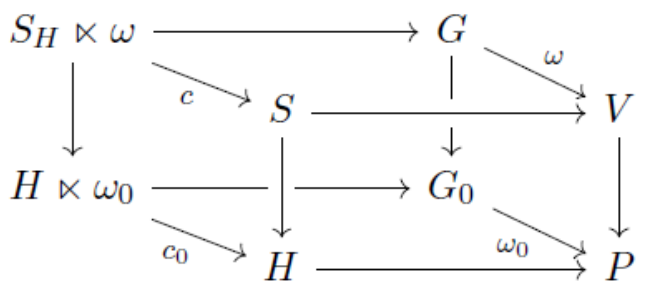

Elements of $S \ltimes\left(\omega, \omega_{0}\right)$ are the squares

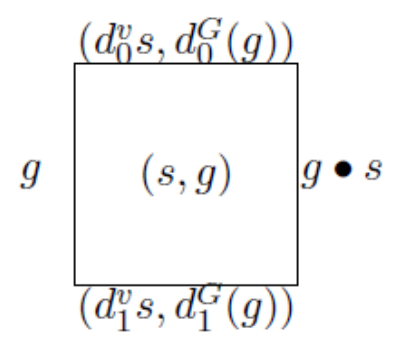

with compositions

$$
\begin{gathered}
\left(s_{1}, g_{1}\right) \circ_{h}\left(s_{2}, g_{2}\right)=\left(s_{1} \circ_{h} s_{2}, g_{1}\right) \\
\left(s_{1}, g_{1}\right) \circ_{v}\left(s_{2}, g_{2}\right)=\left(s_{1} \circ_{v} s_{2}, g_{1} g_{2}\right) .
\end{gathered}
$$

Definition 3.2. A morphism of double groupoids $\phi=\left(\phi_{s}, \phi_{h}, \phi_{v}, \phi_{p}\right):\left(S^{\prime} ; H^{\prime}, V^{\prime} ; P^{\prime}\right) \rightarrow(S ; H, V ; P)$ is called covering morphism associated with the horizontal action if $\left(\phi_{s}, \phi_{v}\right)$ and $\left(\phi_{h}, \phi_{p}\right)$ are covering morphisms of ordinary groupoids.

Let CovDGpd/S denote the category of covering morphisms of double groupoids obtained from horizontal action. The following theorem which is originally given for Lie double groupoids in [19, Theorem 1.7] expresses the categorical equivalence of action double groupoids and corresponding covering morphisms. 
Theorem 3.3. The following equivalence of the categories holds for a double groupoid $S$

$$
\operatorname{CovDGpd} / S \simeq \operatorname{DGpdAct}_{H}(S)
$$

\section{CATEGORICAL EQUIVALENCE BETWEEN ACTIONS AND COVERING MORPHISMS OF DOUBLE GROUP-GROUPOIDS}

In this section we characterize action of a double group-groupoid on a group-groupoid and then obtain covering morphism of double group-groupoids corresponding to the horizontal action. By this way for a given double group-groupoid $S$, we obtain a categorical equivalence between actions and covering morphisms of the double group-groupoid $S$.

A double group-groupoid is defined in [17] to be an internal groupoid in the category of group-groupoids. Therefore a double group-groupoid consists of four related group- groupoids $(S, V),(S, H),(H, P)$ and $(V, P)$ which satisfies the following

$$
\begin{aligned}
& \left(s_{1} \circ_{h} s_{2}\right)+\left(s_{3} \circ_{h} s_{4}\right)=\left(s_{1}+s_{3}\right) \circ_{h}\left(s_{2}+s_{4}\right) \\
& \left(s_{1} \circ_{v} s_{2}\right)+\left(s_{3} \circ_{v} s_{4}\right)=\left(s_{1}+s_{3}\right) \circ_{v}\left(s_{2}+s_{4}\right) .
\end{aligned}
$$

Morphism of double group-groupoids is defined to be double groupoid morphisms such that each function is also group homomorphism. Hence we have a category $D G p G p d$.

Example 4.1. Assume that $G$ is a topological group-groupoid. Then $G$ and $G_{0}$ have topological group structures. So the fundamental groupoids $\pi G$ of $G$ and $\pi G_{0}$ of $G_{0}$ are group-groupoids. Further we have two related groupoids $\left(\pi G, \pi G_{0}\right)$ and $\left(G, G_{0}\right)$. Therefore the quadruple $\left(\pi G ; \pi G_{0}, G ; G_{0}\right)$ becomes a double group-groupoid.

Definition 4.2. For given a group-groupoid $G$, a horizontal action of a double group- groupoid $S=(S ; H, V ; P)$ on group-groupoid morphism $\left(\omega, \omega_{0}\right)$ consists of group homomorphisms $\omega: G \rightarrow V$, $\omega_{0}: G_{0} \rightarrow P$ and a horizontal action of double groupoid on $\left(\omega, \omega_{0}\right)$ such that the following interchange rules hold

i. For given $s_{1}, s_{2} \in S$ and $g_{1}, g_{2} \in G$,

$$
\left(g_{1}+g_{2}\right) \bullet\left(s_{1}+s_{2}\right)=\left(g_{1} \bullet s_{1}\right)+\left(g_{2} \bullet s_{2}\right)
$$

ii. For $x, x_{1} \in G_{0}$ and $h, h_{1} \in H$ we have

$$
\left(x+x_{1}\right) \bullet\left(h+h_{1}\right)=(x \bullet h)+\left(x_{1} \bullet h_{1}\right) .
$$

There is also a similar vertical action of double group-groupoids.

Denote such a horizontal action by $\left(G,\left(\omega, \omega_{0}\right)\right)$ and define a morphism $f:\left(G,\left(\omega, \omega_{0}\right)\right) \rightarrow\left(G^{\prime},\left(\omega^{\prime}, \omega_{0}^{\prime}\right)\right)$ between such actions to be consisting of group-groupoid morphisms $f: G \rightarrow G^{\prime}$ and $f_{0}: G_{0} \rightarrow G_{0}^{\prime}$ with $f(g \bullet s)=f(g) \bullet s$ and $f_{0}(x \bullet h)=f_{0}(x) \bullet h$ such that $\omega^{\prime} f=\omega$ and $\omega_{0}^{\prime} f_{0}=\omega_{0}$. Hence horizontal actions of double group-groupoids and morphisms form a category $\operatorname{DGpGpdAct}_{H}(S)$. 
Theorem 4.3. For given a horizontal action of double group-groupoid $S$ on $\left(\omega, \omega_{0}\right)$, we have a double group-groupoid $\left(S \ltimes \omega ; H \ltimes \omega_{0}, G ; G_{0}\right)$.

Proof: For given a horizontal action of double group-groupoid $S$ on $\left(\omega, \omega_{0}\right)$ we have semidirect product groupoids $S_{H} \ltimes \omega$ and $H \ltimes \omega_{0}$ which are group-groupoids. Therefore $\left(S_{H} \ltimes \omega ; H \ltimes \omega_{0}, G ; G_{0}\right)$ is an action double groupoid with group structure

$$
\left(s_{1}, g_{1}\right)+\left(s_{2}, g_{2}\right)=\left(s_{1}+s_{2}, g_{1}+g_{2}\right) \text {. }
$$

The details are straightforward. Thus the quadruple becomes a double group-groupoid.

We call such a double group-groupoid semidirect product double group-groupoid or action double groupgroupoid with respect to horizontal action of $S$ on $\left(\omega, \omega_{0}\right)$ and denoteby $S \ltimes\left(\omega, \omega_{0}\right)$.

Definition 4.4. A morphism $\phi=\left(\phi_{s}, \phi_{h}, \phi_{v}, \phi_{p}\right):\left(S^{\prime} ; H^{\prime}, V^{\prime} ; P^{\prime}\right) \rightarrow(S ; H, V ; P)$ of double groupgroupoids is called covering morphism associated with the horizontal action if $\left(\phi_{s}, \phi_{v}\right)$ and $\left(\phi_{h}, \phi_{p}\right)$ are covering morphisms of ordinary group-groupoids.

Example 4.5. For given a horizontal action of double group-groupoid $S$ on $\left(\omega, \omega_{0}\right)$, since projections $(c, \omega): S_{H} \ltimes \omega \rightarrow G$ and $\left(c_{0}, \omega_{0}\right): H \ltimes \omega_{0} \rightarrow G_{0}$ are covering morphisms of group-groupoids, the morphism $\phi:\left(S_{H} \ltimes \omega ; H \ltimes \omega_{0}, G ; G_{0}\right) \rightarrow(S ; H, V ; P)$ becomes a covering morphism of double groupgroupoids.

Let CovDGpGpd / $S$ denote the category in which objects are covering morphisms of a double groupgroupoid $S$ and arrows are commutative diagrams of morphisms.

As a result, we have the following categorical equivalence.

Theorem 4.6. The actions and the covering morphisms of double group-groupoid $S$ are categorically equivalent

\section{CovDGpGpd / $S \simeq D G p G p d A c t_{H}(S)$}

Proof: Let $\phi=\left(\phi_{s}, \phi_{h}, \phi_{v}, \phi_{p}\right):\left(S^{\prime} ; H^{\prime}, V^{\prime} ; P^{\prime}\right) \rightarrow(S ; H, V ; P)$ be an object of CovDGpGpd/S. Hence $\left(\phi_{s}, \phi_{v}\right)$ and $\left(\phi_{h}, \phi_{p}\right)$ are covering morphisms of ordinary group-groupoids and then $S_{H}$ acts on $\phi_{v}: V^{\prime} \rightarrow V$ and $H$ acts on $\phi_{p}: P^{\prime} \rightarrow P$. So we have a semidirect product double group-groupoid $\left(S_{H} \ltimes \phi_{v} ; H \ltimes \phi_{p}, V^{\prime} ; P^{\prime}\right)$.

Conversely assume that $\left(G,\left(\omega, \omega_{0}\right)\right)$ is an object of $\operatorname{DGpGpdAct}_{H}(S)$. Then we have an action of $S_{H}$ on $\omega: G \rightarrow V$ and $H$ on $\omega_{0}: G_{0} \rightarrow P$. Hence we have semidirect product group-groupoids $S_{H} \ltimes G$ and $H \ltimes G_{0}$ such that $\left(S_{H} \ltimes G ; H \ltimes G_{0}, G ; G_{0}\right) \rightarrow(S ; H, V ; P)$ is a covering morphism of double groupgroupoids with respect to the horizontal action.

Other details follow and hence omitted. 
Example 4.7. Let $S=(S ; H, V ; P)$ be a double group-groupoid. Then the morphism $\left(1_{s}, 1_{h}, 1_{v}, 1_{p}\right): S \rightarrow S$ is a covering morphism in CovDGpGpd/S which means that $\left(1_{s}, 1_{v}\right)$ and $\left(1_{h}, 1_{p}\right)$ are covering morphism of group-groupoids. The corresponding action is constructed as follows. $S_{H}$ acts on $1_{v}: V \rightarrow V$ and $H$ acts on $1_{p}: P \rightarrow P$ with actions

$$
V \times S_{H} \rightarrow V, v \bullet s=d_{1}^{h}(s) \text { and } d_{0}^{h}(s)=1_{v}(v)
$$

and

$$
P \times H \rightarrow P, p \bullet h=d_{1}^{H}(h) \text { and } d_{0}^{H}(h)=1_{p}(p)
$$

With these actions we have $S_{H} \ltimes 1_{v} \simeq S_{H}$ and $H \ltimes 1_{p} \simeq H$. So we have an action double group-groupoid $\left(S_{H} \ltimes 1_{v} ; H \ltimes 1_{p}, V ; P\right)$ which is isomorphic to $\left(S_{H} ; H, V ; P\right)$.

In below we give an example of covering morphism for double group-groupoids.

Example 4.8. Suppose that $N$ and $M$ are group-groupoids with same base $P$ and $\phi: N \rightarrow M$ is a morphism. Then we have a double group-groupoid $(N \times N ; N, P \times P ; P)$ and an action of $N \times N$ on $\omega: M \rightarrow P \times P$ and of $N$ on $\omega_{0}=1_{p}: P \rightarrow P$ with action $\rho$

$$
\rho: M \times(N \times N) \rightarrow M, \rho(m)\left(n_{1}, n_{2}\right)=\phi\left(n_{1}\right) m \phi\left(n_{2}\right)^{-1}
$$

So semidirect product group-groupoids $(N \times N) \ltimes \omega$ and $N \ltimes 1_{p} \simeq N$ exist. As a result we have an action double group-groupoid $((N \times N) \ltimes \omega ; N, M ; P)$. Elements of $(N \times N) \ltimes \omega$ are triples $\left(n_{1}, m, n_{2}\right)$ of the form

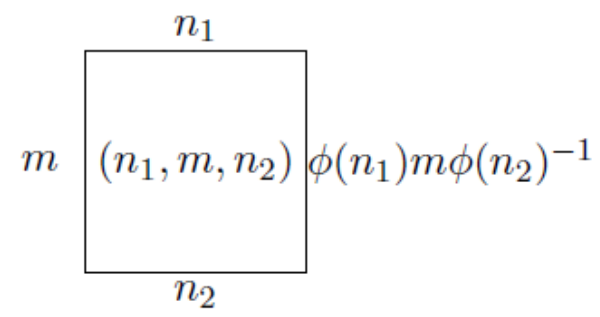

and groupoid compositions defined in [5, Example 1.8] as follows:

$$
\begin{gathered}
\left(n_{1}, m, n_{2}\right) \circ_{h}\left(n_{1}{ }^{\prime}, m^{\prime}, n_{2}{ }^{\prime}\right)=\left(n_{1} n_{1}{ }^{\prime}, m m^{\prime}, n_{2} n_{2}{ }^{\prime}\right) \\
\left(n_{1}, m, n_{2}\right) \circ_{v}\left(n_{1}{ }^{\prime}, m^{\prime}, n_{2}{ }^{\prime}\right)=\left(n_{1}, m m^{\prime}, n_{2}{ }^{\prime}\right) .
\end{gathered}
$$

Group operation of action double group-groupoid is defined by

$$
\left(n_{1}, m, n_{2}\right)+\left(n_{1}{ }^{\prime}, m^{\prime}, n_{2}{ }^{\prime}\right)=\left(n_{1}+n_{1}{ }^{\prime}, m+m^{\prime}, n_{2}+n_{2}{ }^{\prime}\right) \text {. }
$$

Moreover since $(N \times N) \ltimes \omega \rightarrow N \times N$ and $N \ltimes 1_{p} \rightarrow N$ are covering morphism of group-groupoid, $\left((N \times N) \ltimes \omega ; N \ltimes 1_{p}, M ; P\right) \rightarrow(N \times N ; N, P \times P ; P)$ is a covering morphism of double groupgroupoids. 


\section{CONCLUSION}

In this paper we have a categorical equivalence of actions and covering morphisms for double groupgroupoids. Further by [17], double group-groupoids are equivalent to crossed modules over group-groupoids. Hence it could be possible to characterize the concepts of action and covering morphism for such crossed modules. That enables to produce more examples of actions and coverings for double group-groupoids.

\section{ACKNOWLEDGEMENTS}

This paper was presented in the 14th Ankara Mathematics Days Symposium held on June 28-29, 2019.

\section{CONFLICTS OF INTEREST}

No conflict of interest was declared by the authors.

\section{REFERENCES}

[1] Brown, R., Topology and groupoids, BookSurge, LLC, North Carolina, (2006).

[2] Higgins, P. J., Categories and groupoids, Van Nostrand, New York, (1971).

[3] Brown, R., Danesh-Naruie, G. and Hardy, J. P. L., "Topological groupoids. II. Covering morphisms and G-spaces", Math. Nachr., 74: 143-156, (1976).

[4] Brown, R. and Mucuk, O., "Covering groups of nonconnected topological groups revisited", Math. Proc. Cambridge Philos. Soc., 115(1): 97-110, (1994).

[5] Baez, J. C. and Lauda, A. D., "Higher-dimensional algebra V. 2-groups." Theory Appl. Categ., 12: 423-491, (2004).

[6] Brown, R. and Spencer, C. B., "G -groupoids, crossed modules and the fundamental groupoid of a topological group”, Nederl. Akad. Wetensch. Proc. Ser. A, 38(4): 296-302, (1976).

[7] Akiz, H.F., Alemdar, N., Mucuk, O. and Sahan, T., "Coverings of internal groupoids and crossed modules in the category of groups with operations", Georgian Math. J.,20 (2): 223-238, (2013).

[8] Mucuk, O. and Sahan, T., "Covering groupoids of categorical groups", Hacet. J. Math. Stat., 42(4): 419-430, (2013).

[9] Mucuk, O. and Demir, S., “Covering groupoids of categorical rings”, Filomat, 29 (1): 39-49, (2015).

[10] Sahan, T. and Erciyes, A., "Actions of internal groupoids in the category of Leibniz algebras", Commun. Fac. Sci. Univ. Ank. Ser. A1. Math. Stat., 68 (1): 619-632, (2019).

[11] Ehresmann, C., "Catégories doubles et categories structures", C. R. Acad. Sci. Paris, 256:1198-1201, (1963).

[12] Ehresmann, C., “Catégories structurées”, Ann. Sci. École Norm. Sup., 80 (3): 349-426, (1963).

[13] Brown, R., Higgins, P. J. and Sivera, R., Nonabelian algebraic topology. filtered spaces, crossed complexes, cubical homotopy groupoids, European Mathematical Society Tracts in Mathematics 15, (2011). 
[14] Brown, R. and Spencer, C. B., "Double groupoids and crossed modules", Cahiers Topologie Géom. Différentielle, 17(4): 343-362, (1976).

[15] Whitehead, J. H. C., "Note on a previous paper entitled on adding relations to homotopy groups", Ann. of Math., 47(2): 806-810, (1946).

[16] Whitehead, J. H. C., “Combinatorial homotopy II”, Bull. Amer. Math. Soc., 55: 453-496, (1949).

[17] Temel S., Sahan T. and Mucuk O., "Crossed modules, double group-groupoids and crossed squares", arXiv: 1802.03978, submitted for publication, (2018).

[18] Mucuk, O., and Demir, S., "Normality and quotient in crossed modules over groupoids and double groupoids", Turkish J. Math., 42 (5): 2336-2347, (2018).

[19] Brown, R. and Mackenzie, K. C. H., "Determination of a double Lie groupoid by its core diagram", J. Pure Appl. Algebra, 80(3): 237-272, (1992).

[20] Mackenzie, K., Lie groupoids and Lie algebroids in differential geometry, London Mathematical Society Lecture Note Series, 124. Cambridge University Press, Cambridge, (1987). 